OPEN ACCESS

Edited by:

Ashish Saxena,

Weill Cornell Medical Center,

United States

Reviewed by:

Giuseppe Galletti,

Weill Cornell Medical Center,

United States

Jelena Stojsic,

University of Belgrade,

Serbia

${ }^{*}$ Correspondence:

Shiyue $L i$

lishiyue@188.com

Chengzhi Zhou

zhouchengzhi@gird.cn

${ }^{+}$These authors have contributed equally to this work

Specialty section: This article was submitted to

Thoracic Oncology,

a section of the journal

Frontiers in Oncology

Received: 26 February 2021 Accepted: 26 April 2021

Published: 14 May 2021

Citation:

Xie $X$, Wang L, Wang $X$,

Fan $W-H$, Qin $Y$, Lin $X$,

Xie Z, Liu M, Ouyang $M$,

LiS and Zhou C (2021)

Evaluation of Cell Surface

Vimentin Positive Circulating

Tumor Cells as a Diagnostic

Biomarker for Lung Cancer

Front. Oncol. 11:672687. doi: 10.3389/fonc.2021.672687

\section{Evaluation of Cell Surface Vimentin Positive Circulating Tumor Cells as a Diagnostic Biomarker for Lung Cancer}

\author{
Xiaohong Xie ${ }^{1 \dagger}$, Liqiang Wang ${ }^{1 \dagger}$, Xinni Wang ${ }^{1 \dagger}$, Wan-Hung Fan ${ }^{2}$, Yinyin Qin ${ }^{1}$, \\ Xinqing Lin ${ }^{1}$, Zhanhong Xie ${ }^{1}$, Ming Liu ${ }^{1}$, Ming Ouyang ${ }^{1}$, Shiyue $L_{i}{ }^{1 *}$ and Chengzhi Zhou ${ }^{1 *}$ \\ 1 Department of Pulmonary and Critical Care Medicine, Guangzhou Institute of Respiratory Health, State Key Laboratory of \\ Respiratory Disease, National Clinical Research Center for Respiratory Disease, The First Affiliated Hospital of Guangzhou \\ Medical University, Guangzhou, China, ${ }^{2}$ Department of Clinical Medical Affairs, Hangzhou Watson Biotech, Hangzhou, China
}

Background: Circulating tumor cells (CTCs) represent a collection of heterogeneous cells. Studies have shown epithelial CTCs and folate receptor (FR) positive CTCs could be used as diagnostic biomarkers for lung cancer (LC). This study aimed to determine whether cell surface vimentin (CSV) positive CTCs could be used as a biomarker for LC as well.

Methods: 78 treatment-naïve non-small-cell lung cancer (NSCLC) patients, 21 patients with benign lung diseases (BLD) and 9 healthy donors (HD) were enrolled in this study. CTC detection was performed using CytoSorter ${ }^{\circledR}$ mesenchymal CTC kit (CSV). The correlation between CSV positive CTCs (CSV-CTCS) and LC patients' clinicopathological characteristics would be evaluated, and diagnostic performances of CSV-CTCs and serum tumor markers for LC would be compared.

Results: CTC detection rates (average CTC count: range) in LC patients, patients with BLD and HD were 83.33\% (2.47: 0-8), 47.62\% (0.5: 0-3) and 0\% (0: 0), respectively. CSVCTCs could be used to differentiate LC patients from the patients with BLD and HD ( $P<$ 0.0001). CSV-CTCs were correlated with cancer stage, lymph node involvement and distant metastasis ( $P=0.0062,0.0014$ and 0.0021 , respectively). With a CTC cut-off value of 2, CSV-CTCs would have a sensitivity and specificity of 0.67 and 0.87 , respectively, for diagnosing LC. CSV-CTC positive rates showed statistical differences among HD, BLD patients and LC patients at different cancer stages $(P<0.0001)$. Furthermore, CSV-CTC positive rates were positively correlated with tumor size, lymph node involvement and distant metastasis ( $P=0.0163,0.0196$ and 0.03 , respectively). CSV-CTCs had a better diagnostic performance than serum tumor makers, such as carcinoembryonic antigen (CEA), neuron-specific enolase (NSE), cancer antigen 125 (CA125) and CA153. 


\begin{abstract}
Conclusion: When CTC cut-off is set to 2 CTCs per $7.5 \mathrm{~mL}$ of blood, CSV-CTCs can be considered as an acceptable biomarker for diagnosing $L C$ with a sensitivity and specificity of 0.67 and 0.87 , respectively.
\end{abstract}

Keywords: circulating tumor cells, cell surface vimentin (CSV), NSCLC, cancer diagnosis, serum tumor markers

\section{INTRODUCTION}

Lung cancer (LC) is the most common cancer and the leading cause of cancer-related deaths both worldwide and in China (1, 2). There were approximately 787,000 newly diagnosed cases and 631,000 deaths for LC in 2015 in China (3). China occupies only about $20 \%$ of the world's population, but more than one third of the LC cases are in China. Although diagnostic and treatment modalities for LC have an enormous progress in recent years, most LC patients still have a poor prognosis with a 5-year survival rate ranging from $4-17 \%$ depending on cancer stage and regional differences (4). "Early detection, early treatment" means that patients would have a better treatment strategy and survival outcome if the tumors were diagnosed earlier (5). However, most LC patients are already in advanced stages of disease at the time of diagnosis. Thus, early detection of LC is important to improve the overall survival.

The primary approaches to diagnose LC include medical imaging examination, serum tumor markers test and biopsy. Tumors are usually quite small at an early stage and therefore they can be hardly detected by the imaging techniques due to the sensitivity limitation. Biopsy is the gold standard for cancer diagnosis, but it cannot be performed regularly due to the invasive nature, thus it cannot be used as a surveillance means to monitor the real-time progression of disease. Serum tumor makers, such as neuron-specific enolase (NSE), carcinoembryonic antigen (CEA), cancer antigen 125 (CA125) and CA153, are frequently used in practice for LC diagnosis (6). However, serum tumor makers generate easily false positive results due to inflammations, infections, pregnancy, or other physical conditions, rendering these markers not very trustworthy. As a consequence, to improve the clinical outcomes of LC patients, it is in need to find a reliable biomarker for better screening and early diagnosis of LC.

Circulating tumor cells (CTCs) are tumor cells that have detached from the primary tumor or metastatic lesions and escaped into circulation. CTCs can colonize other organ to give rise to a new metastatic lesion once they find a suitable site (7). Studies have suggested that CTCs represent the undergoing process of metastasis and can be used as a prognostic marker to predict clinical outcomes of LC patients (8-11). CTCs can be detected in the blood even when the tumor is clinically undetectable (typically $<0.01 \mathrm{~cm}^{3}$ ) (12), implying that CTC is a good biomarker candidate for early diagnosis of LC.

CTCs are a collection of heterogeneous cells, indicating that each CTC may differ from each other in term of cell size, gene mutation and protein expression $(13,14)$. Several studies have suggested the use of CTCs for screening and early diagnosis of
LC (15-17). Duan et al. used GILUPI CellCollector to detect CTCs in 44 patients suspected of LC and in 20 healthy donors (HD). With a CTC cut-off of 1 , CellCollector has a sensitivity and specificity of 0.53 and 0.9 , respectively (15). Li et al. used a negative enrichment-fluorescence in situ hybridization (NEFISH) method to detect CTC in 174 LC patients and 90 control and discovered that NE-FISH had a sensitivity and specificity of 0.68 and 1 , respectively (16). Chen et al. used ligand-targeted polymerase chain reaction (LT-PCR) technique to detect folate receptor (FR) positive CTCs in 756 participants, including 473 patients with non-small cell lung cancer (NSCLC), 227 patients with benign lung diseases (BLD), and $56 \mathrm{HD}$, and found that with a sensitivity and specificity of 0.76 and 0.88 , respectively, FR positive CTCs could be used as a biomarker in the diagnosis of NSCLC (17).

Both epithelial and FR positive CTCs can be used as biomarkers for LC diagnosis (15-17), we would like to know whether cell surface vimentin (CSV) positive CTCs (CSV-CTCs) can be used as a biomarker for LC as well. 78 NSCLC patients, 21 patients with BLD and $9 \mathrm{HD}$ were recruited in this study. The diagnostic performances of CSV-CTCs and serum tumor markers for LC would be compared and the correlation between CSV-CTCs and LC patients' clinicopathological characteristics would be analyzed.

\section{MATERIALS AND METHODS}

\section{Ethics and Participants}

In total, 78 NSCLC patients, including 30 stage I, 7 stage II, 13 stage III and 28 stage IV, 21 patients with BLD and 9 HD were enrolled in the First Affiliated Hospital of Guangzhou Medical University. The included patients were diagnosed between May 2019 and October 2019. All included patients had negative history of malignancy within 5 years prior to enrollment, and were treatment-naïve before enrollment. Recruited patients with BLD suffered from hamartoma, papilloma, granulomatous inflammation, fibroma, benign nodule and other lung infections. All included HD had no abnormal finding in medical imaging examination and no medical history of any malignant disease. The LC patient demographics and clinical information, including age, gender, smoking history, tumor histology, TNM stage, and serum levels of NSE, CEA, CA125 and CA153 were collected.

\section{Identification of CSV-CTCs}

CytoSorter $^{\circledR}$ (Hangzhou Watson Biotech, Hangzhou, China) CSV mesenchymal CTC kit was used for CTC detection. CTC detection procedure was following the manufacturer protocol 
and was described in the previous study (18). In brief, the streptavidin-functionalized CytoChipNano was first coated with biotin-labeled CSV antibody before placing onto CytoSorter $^{\circledR}$. $7.5 \mathrm{~mL}$ of collected peripheral blood was first proceeded to gradient-centrifuge within 6 hours after collection to collect the peripheral blood mononuclear cells (PBMC). PBMC sample solution was then transferred into the spiral sample tube on CytoSorter ${ }^{\circledR}$. The enrichment procedure was controlled by CytoSorter ${ }^{\circledR}$ software designed for each CTC capturing antibody. Once the CTC enrichment was finished, the CytoChipNano was removed from CytoSorter ${ }^{\circledR}$ and proceeded to the manual immunofluorescence staining of CSV-FITC (fluorescein isothiocyanate), CD45-PE (lymphocyte antigen-phycoerythrin) and DAPI (4,6-diamidino-2phenylindole). An OPPNO immunofluorescence microscope (DSY5000X, OPPNO, Chongqing, China) was used to identify CTCs by searching for CSV-FITC positive, CD45-PE negative, and DAPI positive cells. CSV-CTCs appeared green and blue, but not orange, while white blood cells appeared orange and blue, but not green, under florescent microscope. All identified cells must be checked for morphology under bright-field.

\section{Measurement of Serum Tumor Markers}

A $3 \mathrm{~mL}$ of fasting venous blood sample was collected from each patient and HD in the morning. Serum was separated by centrifugation at $4000 \mathrm{rpm}$ for 10 minutes within 2 hours after blood collection. NSE, CEA, CA125 and CA153 were detected by an automatic electrochemical luminescence analyzer (Cobas e602, Roche, Germany). All serum tumor marker tests were conducted according to instrument operating manuals. $17.5 \mathrm{ng} / \mathrm{mL}, 5 \mathrm{ng} /$ $\mathrm{mL}, 35 \mathrm{U} / \mathrm{mL}$ and $25 \mathrm{U} / \mathrm{mL}$ were considered as the upper limits of normality for NSE CEA, CA125 and CA153, respectively.

\section{Statistical Analysis}

Statistical analyses were performed using Prism 6.0 (Graphpad, La Jolla, CA, USA) and SPSS 20 (IBM Corp., Armonk, NY, USA). The $\mathrm{x}^{2}$ test and Fisher's exact test were used for the comparison of categorical parameters. One-way analysis of variance (ANOVA) was performed to calculate the differences among multiple groups. Student $t$ test was used for continuous variables, as appropriate. The diagnostic performance was evaluated by the receiver operating characteristic (ROC) curve according to the value of sensitivity, specificity and area under curve (AUC). CTC cut-off value was determined by the highest Youden index (sensitivity + specificity - 1). Comparison of diagnostic potency of different systems was rated by the AUC value. A two-sided p value less than 0.05 was considered statistically significant.

\section{RESULTS}

\section{Detection of CSV-CTCs in LC Patients, Patients With BLD and HD}

A CSV-CTC is shown in Figure 1A as a cell appearing green and blue, but not orange under florescent microscope. CSV-CTCs were detected in 65 out of $78 \mathrm{LC}$ patients with a mean of 2.47 cells (range:0-8), 10 out of 21 patients with BLD with a mean of 0.5 cells (range: $0-3$ ) and in none of the $9 \mathrm{HD}$ as shown in Table 1. A significant difference of CSV-CTCs was found among LC patients, patients with BLD and $\operatorname{HD}(P<0.0001$, Figure 1B). Furthermore, if LC patients were broken-down by stage, significant differences of CSV-CTCs were still found between BLD patients and stage I, II, III or IV LC patients $(P=0.0167$, $0.0307,0.0014$, or $<0.0001$, Figure 1C), indicating that CSVCTCs could be used as a biomarker to distinguish LC patients from the patients with BLD and HD. 9 out of 10 patients with BLD who were found to have CSV positive cells had either inflammation diseases, fibrosis or other lung infection conditions.

\section{CSV-CTCs Are Associated With Lymph Node and Distant Metastases in LC}

CSV-CTC enumeration in stage I-IV LC patients ranged from 0 to 4 CTCs per $7.5 \mathrm{~mL}$ of blood (mean: 1.6), 0-4 (2), 0-5 (2.54) and $0-8$ (3.5), respectively $(P=0.0062$, Figure 1C). CTC detection rates were $73.33 \%, 85.71 \%, 84.62 \%$ and $92.86 \%$, respectively. CSV-CTCs are positively correlated with cancer stage. Patients aged over 60 years old had a slightly higher CTC detection rate (90\% compared to $76.32 \%)$ and more CTCs (2.88 compared to

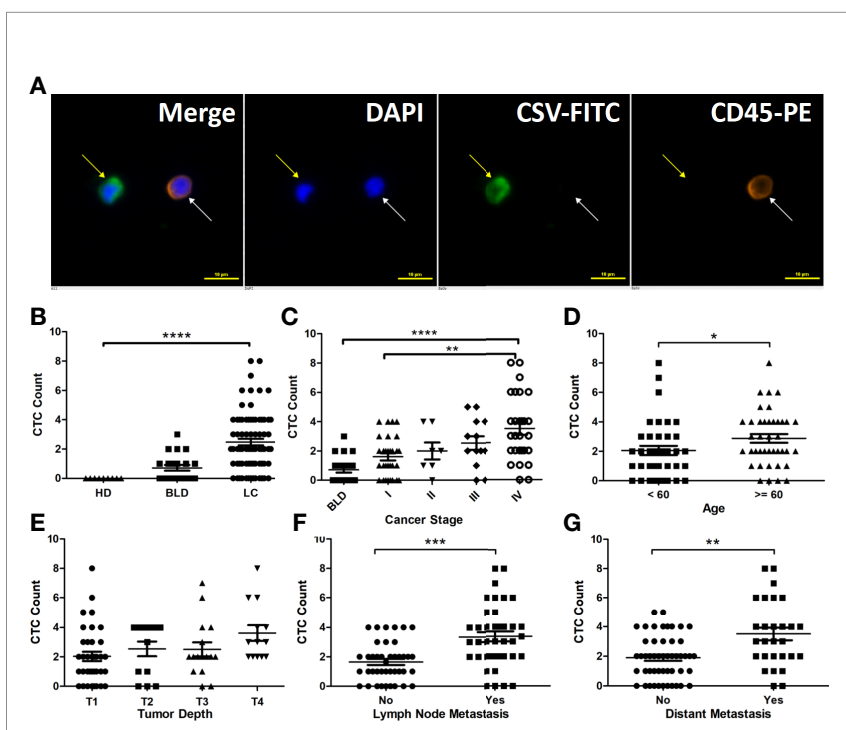

FIGURE 1 | CSV-CTCs are correlated with LC patients ' cancer stages, lymph node and distant metastases and can be used to distinguish LC patients from patients with BLD and HD. (A) Immunofluorescent staining of a captured CSV-CTC and a white blood cell (WBC), indicated by the yellow and white arrows, respectively. CSV positive CTCs are defined as DAPI (blue) positive, CSV (FITC, green) positive and CD45 (PE, orange) negative cells, while a WBC as a DAPI positive, CD45 positive and CSV negative cell. Scale bar represents $10 \mu \mathrm{m}$, immunofluorescent staining, X 20 (B) CSV-CTC enumeration can differentiate LC patients from BLD patients and HD (both $P<0.0001)$. (C) CSV-CTCs are correlated with cancer stage $(P=0.0062)$. Significant differences of CSV-CTCs are found between BLD patients and stage I, II, III or IV LC patients ( $P=0.0167,0.0307,0.0014$, or $<0.0001)$. CSV-CTCs are correlated as well with age $(P=0.0274)$, lymph node metastasis $(P=0.0002)$ and distant metastasis $(P=0.0021)$, as shown in (D, F, G), respectively. However, CSV-CTCs are not associated with tumor

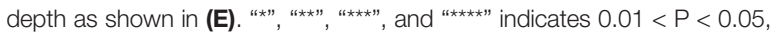
$0.001<P<0.01,0.0001<P<0.001$, and $P<0.0001$, respectively. 
TABLE 1 | CTCs can be used to distinguish lung cancer patients from the control (HD + BLD patients).

\begin{tabular}{|c|c|c|c|c|c|c|}
\hline Group & $\mathbf{n}$ & Average (Median) Age (years) & CTCs $\geq 1$ (per $7.5 \mathrm{~mL}$ ) & CTC Detection Rate (\%) & Average CTC Count (Range) & $P$ \\
\hline BLD & 21 & 51.57 (55) & 10 & 47.62 & $0.5(0-3)$ & \\
\hline LC & 78 & $58.55(60)$ & 65 & 83.33 & $2.47(0-8)$ & \\
\hline BLD & 21 & $51.57(55)$ & 10 & 47.62 & $0.5(0-3)$ & \\
\hline TNM I & 30 & $55.17(55.5)$ & 22 & 73.33 & $1.6(0-4)$ & \\
\hline TNM ॥ & 7 & $60.29(60)$ & 6 & 85.71 & $2(0-4)$ & \\
\hline
\end{tabular}

CTCS, circulating tumor cells; HD, healthy donor; BLD, benign lung disease; n, number; LC, lung cancer; TNM, tumor-node-metastasis.

Bold values mean statistically significant. Bold values are all less than 0.05 .

2.05) than patients younger than 60 years old $(P=0.0274$,

Figure 1D), which might be due to that the former had usually advanced tumors. CSV-CTCs are statistically associated with lymph node involvement and distant metastasis $(P=0.0014$ and 0.0021, respectively, Table 2). Patients with lymph node or distant metastasis had more CTCs (3.34 versus 1.65 or 3.5 versus 1.9, Figures 1F, G). No significant difference of CSVCTCs was found among LC patients grouped by gender, smoking history or tumor type (adenocarcinoma or squamous) as shown in Table 2. CSV-CTCs were not associated with tumor depth $(P=0.0646$, Figure 1E). Taken together, our results show that
CSV-CTCs are correlated with lymph node and distant metastases, suggesting that the CSV-CTCs represent the CTC sub-population with more invasive nature.

\section{Evaluation of Diagnostic Performance of CSV-CTCs for LC}

CSV-CTCs were detected in almost half of the enrolled patients with BLD (47.62\%). In order to reduce the false positives in BLD patients, a ROC curve was drawn and Youden index was calculated as shown in Figure $\mathbf{2}$ and Table 3 to determine the CTC cut-off at which CSV-CTCs would have the best diagnostic

TABLE 2 | Correlation of CTCs with LC patients' demographics and clinical characteristics.

\begin{tabular}{|c|c|c|c|c|c|c|}
\hline Characteristics & $\mathbf{n}$ & Average (Median) Age (years) & CTCs $\geq 1$ (per $7.5 \mathrm{~mL}$ ) & CTC Detection Rate (\%) & Average СТC Count (Range) & $P$ \\
\hline \multicolumn{7}{|l|}{ Gender } \\
\hline Male & 43 & $59.4(60)$ & 35 & 81.4 & $2.56(0-8)$ & 0.7398 \\
\hline \multicolumn{7}{|l|}{ Age } \\
\hline$\geq 60$ & 40 & $65.15(65)$ & 36 & 90 & $2.88(0-8)$ & 0.0274 \\
\hline$<60$ & 38 & $51.61(52)$ & 29 & 76.32 & $2.05(0-8)$ & \\
\hline \multicolumn{7}{|l|}{ Smoking History } \\
\hline \multicolumn{7}{|l|}{ Histology } \\
\hline Adenocarcinoma & 65 & $58.63(60)$ & 55 & 84.62 & $2.42(0-8)$ & 0.5576 \\
\hline Squamous & 13 & $58.15(58)$ & 10 & 76.92 & $2.77(0-8)$ & \\
\hline \multicolumn{7}{|l|}{ TNM Stage } \\
\hline । & 30 & $55.17(55.5)$ & 22 & 73.33 & $1.6(0-4)$ & 0.0062 \\
\hline$\|$ & 7 & $60.29(60)$ & 6 & 85.71 & $2(0-4)$ & \\
\hline T2 & 13 & $59.92(61)$ & 10 & 76.92 & $2.54(0-4)$ & \\
\hline T3 & 16 & $59.81(60)$ & 14 & 87.5 & $2.5(0-7)$ & \\
\hline $\mathrm{T} 4$ & 13 & $63.15(64)$ & 13 & 100 & $3.62(2-8)$ & \\
\hline \multicolumn{7}{|c|}{ Lymph Node Involvement } \\
\hline NO & 40 & $56.88(56.5)$ & 31 & 77.5 & $1.65(0-4)$ & 0.0014 \\
\hline N1 & 4 & $61.5(62.5)$ & 4 & 100 & $4.5(3-6)$ & \\
\hline N2 & 19 & $61.89(63)$ & 17 & 89.47 & $3.26(0-8)$ & \\
\hline N3 & 15 & $58(60)$ & 13 & 86.67 & $3.13(0-8)$ & \\
\hline \multicolumn{7}{|c|}{ Lymph Node Metastasis } \\
\hline Yes & 38 & $60.32(61)$ & 34 & 89.5 & $3.34(0-8)$ & 0.0002 \\
\hline No & 40 & $56.88(56.5)$ & 31 & 77.5 & $1.65(0-4)$ & \\
\hline \multicolumn{7}{|l|}{ Distant Metastasis } \\
\hline MO & 50 & $57.5(57.5)$ & 39 & 78 & $1.9(0-5)$ & 0.0021 \\
\hline
\end{tabular}

CTCS, circulating tumor cells; $n$, number; LC, lung cancer; TNM, tumor-node-metastasis. Bold values mean statistically significant. Bold values are all less than 0.05 . 

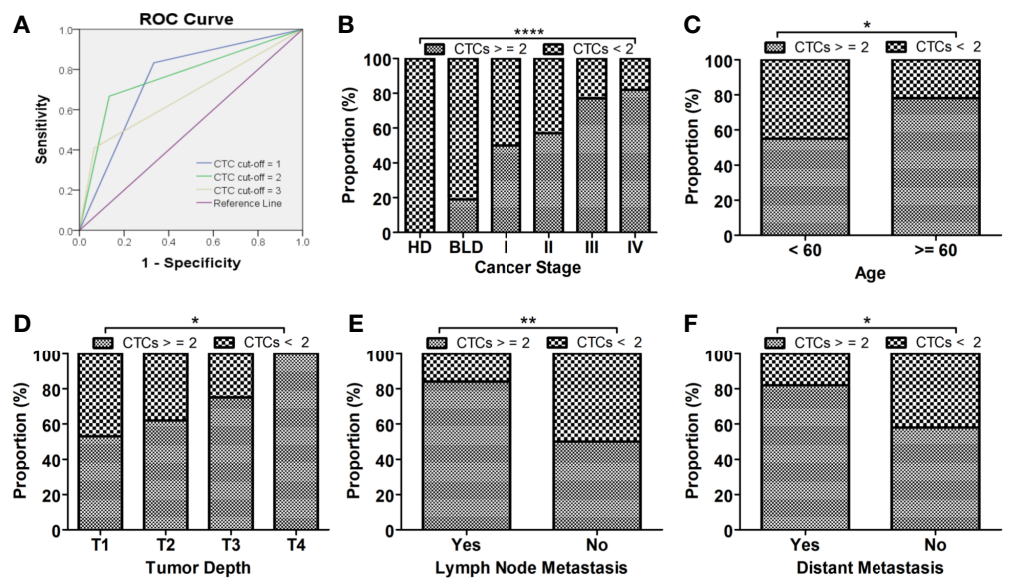

FIGURE 2 | CSV-CTCs can be used as biomarker for diagnosing LC. (A) ROC curves of CSV-CTCs for LC with different CTC cut-off. When CTC cut-off value is set to 2 , the ROC curve has the highest AUC of 0.767 with a sensitivity and specificity of 0.67 and 0.87 , respectively. (B) CSV-CTC positive rates show significant differences among LC patients, patients with BLD and HD $(P<0.0001)$. CSV-CTC positive rates show significant differences as well between BLD and stage I, III or IV LC patients $(P=0.0389,0.0014$ or $<0.0001)$. CSV-CTC positive rates are associated with LC patients' age, tumor depth, lymph node and distant metastases

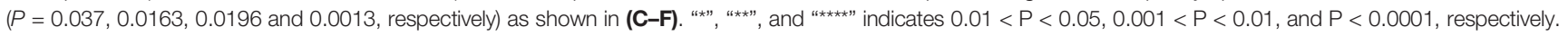

performance for LC. When CTC cut-off was set to 1 or 2 , it generated a sensitivity and specificity of 0.83 and 0.67 , or 0.67 and 0.87 , respectively (Table 3). Youden index of CTC cut-off of 2 is slightly higher than that of 1 ( 0.53 versus 0.5 , Table 3$)$. If CTC positive was defined as any patients with CTCs no less than 2, CSV-CTC positive rates showed a significant difference among LC patients, patients with BLD and HD as shown in Table $4(P<$ 0.0001) and Figure 2B. If LC patients were broken-down by stage, CSV-CTC positive rates showed significant differences between BLD and stage I, III or IV LC patients $(P=0.0389$, 0.0014 or $<0.0001$, Figure 2B). However, the CSV-CTC positive rate did not show a significant difference between BLD and stage II LC patients $(P=0.1423)$, which might be due to the small sample size of enrolled stage II patients $(n=7)$.

\section{Correlation of CSV-CTC Positive Rates With LC Patients' Clinicopathological Characteristics}

CSV-CTC positive rates were correlated with age, lymph node involvement, lymph node and distant metastases $(P=0.037$, 0.0196, 0.0013 and 0.03, respectively, Table 5 and Figures 2C, E, F). CTC positive rates were not correlated with gender, smoking

TABLE 3 | Youden index of different CTC Cut-off values.

\begin{tabular}{|c|c|c|c|c|}
\hline CTC cut-off (per $7.5 \mathrm{~mL}$ ) & Sensitivity & Specificity & Youden index & Area Under Curve (AUC) \\
\hline 1 & 0.83 & 0.67 & 0.5 & 0.75 \\
\hline 2 & 0.67 & 0.87 & 0.53 & 0.767 \\
\hline 3 & 0.41 & 0.97 & 0.38 & 0.672 \\
\hline
\end{tabular}

CTC, circulating tumor cell.

TABLE 4 | CTCs can be used to distinguish LC patients from the control (BLD patients + HD) when CTC cut-off is set to 2.

\begin{tabular}{|c|c|c|c|c|c|c|}
\hline \multirow[t]{2}{*}{ Group } & \multirow[t]{2}{*}{$\mathbf{n}$} & \multicolumn{2}{|c|}{ CTCs $\geq 2$ (per $7.5 \mathrm{~mL})$} & \multicolumn{2}{|c|}{ CTCs < 2 (per 7.5 mL) } & \multirow[t]{2}{*}{$\mathbf{P}$} \\
\hline & & $\mathbf{n}$ & Proportion (\%) & $\mathbf{n}$ & Proportion (\%) & \\
\hline HD & 9 & 0 & 0.00 & 9 & 100.00 & $<0.0001$ \\
\hline BLD & 21 & 4 & 19.05 & 17 & 80.95 & \\
\hline LC & 78 & 52 & 66.67 & 26 & 33.33 & \\
\hline HD & 9 & 0 & 0.00 & 9 & 100.00 & $<0.0001$ \\
\hline BLD & 21 & 4 & 19.05 & 17 & 80.95 & \\
\hline TNM I & 30 & 15 & 50.00 & 15 & 50.00 & \\
\hline TNM ॥ & 7 & 4 & 57.14 & 3 & 42.86 & \\
\hline TNM III & 13 & 10 & 76.92 & 3 & 23.08 & \\
\hline TNM IV & 28 & 23 & 82.14 & 5 & 17.86 & \\
\hline
\end{tabular}

CTCS, circulating tumor cells; LC, lung cancer; HD, healthy donor; BLD, benign lung disease; $n$, number; TNM, tumor-node-metastasis.

Bold values mean statistically significant. Bold values are all less than 0.05 . 
TABLE 5 | Relationship of CTCs with LC patients' demographics and clinical characteristics when CTC cut-off is set to 2.

\begin{tabular}{|c|c|c|c|c|c|}
\hline Characteristics & N (Total = 78) & Proportion (\%) & CTCs $\geq 2$ (per $7.5 \mathrm{~mL})$ & CTCs < 2 (per $7.5 \mathrm{~mL})$ & $P$ \\
\hline \multicolumn{6}{|l|}{ Gender } \\
\hline Male & 43 & 55.13 & 29 & 14 & 0.8719 \\
\hline Female & 35 & 44.87 & 23 & 12 & \\
\hline \multicolumn{6}{|l|}{ Age } \\
\hline$\geq 60$ & 40 & 51.28 & 31 & 9 & 0.037 \\
\hline$<60$ & 38 & 48.72 & 21 & 17 & \\
\hline \multicolumn{6}{|l|}{ Smoking History } \\
\hline Yes & 35 & 44.87 & 26 & 9 & 0.1979 \\
\hline No & 43 & 55.13 & 26 & 17 & \\
\hline \multicolumn{6}{|l|}{ Histology } \\
\hline Adenocarcinoma & 65 & 83.33 & 42 & 23 & 0.3903 \\
\hline Squamous & 13 & 16.67 & 10 & 3 & \\
\hline \multicolumn{6}{|l|}{ TNM Stage } \\
\hline 1 & 30 & 38.46 & 15 & 15 & 0.0533 \\
\hline$\|$ & 7 & 8.97 & 4 & 3 & \\
\hline III & 13 & 16.67 & 10 & 3 & \\
\hline IV & 28 & 35.90 & 23 & 5 & \\
\hline \multicolumn{6}{|l|}{ Tumor Depth } \\
\hline T1 & 36 & 46.15 & 19 & 17 & 0.0163 \\
\hline $\mathrm{T} 2$ & 13 & 16.67 & 8 & 5 & \\
\hline Т3 & 16 & 20.51 & 12 & 4 & \\
\hline $\mathrm{T} 4$ & 13 & 16.67 & 13 & 0 & \\
\hline \multicolumn{6}{|c|}{ Lymph Node Involvement } \\
\hline NO & 40 & 51.28 & 20 & 20 & 0.0196 \\
\hline N1 & 4 & 5.13 & 4 & 0 & \\
\hline N2 & 19 & 24.36 & 16 & 3 & \\
\hline N3 & 15 & 19.23 & 12 & 3 & \\
\hline \multicolumn{6}{|c|}{ Lymph Node Metastasis } \\
\hline Yes & 38 & 48.72 & 32 & 6 & 0.0013 \\
\hline No & 40 & 51.28 & 20 & 20 & \\
\hline \multicolumn{6}{|l|}{ Distant Metastasis } \\
\hline MO & 50 & 64.10 & 29 & 21 & 0.03 \\
\hline M1 & 28 & 35.90 & 23 & 5 & \\
\hline
\end{tabular}

CTCS, circulating tumor cells; $n$, number; LC, lung cancer; TNM, tumor-node-metastasis. Bold values mean statistically significant. Bold values are all less than 0.05.
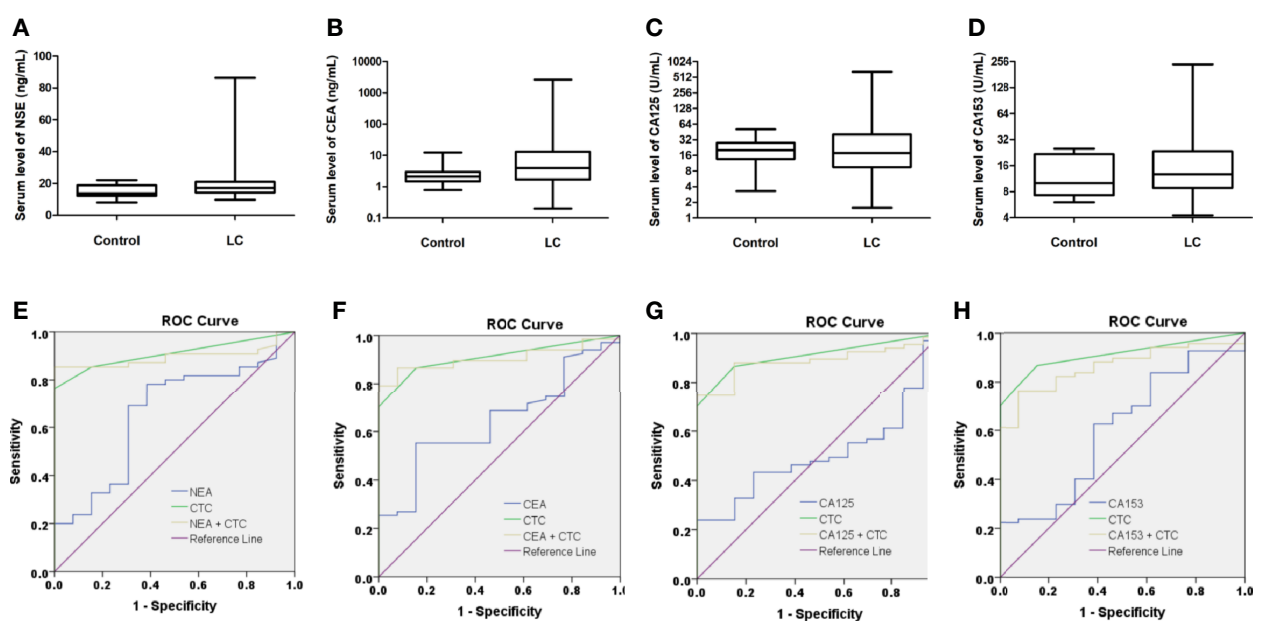

FIGURE 3 | Serum levels of tumor biomarkers in control (BLD + HD) and LC patients. Serum levels of neuron-specific enolase (NSE), carcinoembryonic antigen (CEA), cancer antigen 125 (CA125) and CA153 in control and LC patients are shown in (A-D). None of these serum biomarkers showed statistical significance in differentiating LC patients from the control. (box plot with mean, min to max). Conjugation of CTCs with serum markers did not improve the diagnostic performances for LC, since all AUC of combinations of CTCs with serum tumor markers were reduced as compared to CTCs alone as shown in (E-H). 
history, nor tumor histologic type as shown in Table 5. CTC positive rates was not associated with cancer stages $(P=0.0533$, Table 5), although CTC positive rates did increase in more advanced LC. It might be due to the small sample size. CSV-CTC positive rate was correlated with tumor depth $(P=0.0163$, Table 5).

\section{Comparison of CSV-CTCs and Serum Tumor Markers for Diagnosing LC}

67 LC patients and 13 control donors (9 patients with BLD and 4HD) had serum tumor marker tests data at enrollment. Serum levels of tumor markers, including NSE, CEA, CA125, and CA153, in LC patients and control are shown in Figures 3A-D. No statistical difference of serum tumor marker level between LC patients and control was found, indicating that serum tumor markers were not good biomarkers for LC. However, when we compared serum tumor markers with LC patients' clinicopathological characteristics, most serum tumor makers were correlated with cancer stage, tumor size, lymph node involvement and distant metastasis as shown in Table 6. Most serum tumor markers were not associated with gender, age, smoking history or tumor histologic type, except for CA153, which was correlated with age and tumor type $(P=0.0291$ and 0.0081 , respectively, Table 6). Serum tumor markers could reflect tumor burden in LC. LC patients at advanced cancer stage, with bigger tumors, lymph node or distant metastases, tend to have higher serum level of tumor markers. Serum tumor markers are associated as well with CSV-CTC status as shown in Table 6. Significant differences of NSE, CEA and CA153 levels between patients with and without CSVCTCs were found ( $P=0.0063,0.0191$ and 0.0067 , respectively). Significant differences of serum levels of CEA, CA125 and CA153 were found between CSV-CTC positive and negative patients $(P=$ $0.0202,0.0315$ and 0.0279 , respectively). Among the 4 serum tumor markers, CA153 is the tumor marker most related with LC patients' clinicopathological characteristics.

ROC curves were drawn to compare the diagnostic performance of CSV-CTCs with serum tumor makers. The diagnostic efficacy of CSV-CTCs (AUC $=0.909$ ) was significantly higher than those of NSE, CEA, CA125 and CA153 (AUC $=0.661,0.673,0.53$, and 0.624, respectively, Figures 3E-H). Combinations of CTCs with serum tumor markers for diagnosing LC were also explored. However, the combination did not improve the diagnostic potency, for all AUC of combinations were reduced as compared to CTCs alone (Figures 3E-H).

\section{DISCUSSION}

Studies have suggested the use of CTCs for LC screening (15-17, 19). However, the low detection rate restricts the clinical application of CTCs as a diagnostic aid in practice. CellSearch $^{\circledR}$, the only CTC system that has been cleared by the US Food and Drug Administration (FDA) for clinical use in patients with metastatic breast, colorectal and prostate cancer (20-22), utilizes anti-EpCAM (epithelial cell adhesion molecule) immunomagnetic beads to capture epithelial CTCs. Krebs et al. used CellSearch ${ }^{\circledR}$ to detect CTCs in stage III and IV LC patients, and the CTC detection rates were only $5 \%$ and $32 \%$, respectively (23). Marchetti et al. used CellSearch ${ }^{\circledR}$ and CTCs were detected in 15 out of 41 NSCLC patients (41\%) (24). Ilie et al. used CellSearch ${ }^{\circledR}$ and ISET $^{\circledR}$ to detect CTCs in advanced NSCLC patients, and the CTC detection rates were $32 \%$ and $76 \%$, respectively (25). Many platforms have been developed to isolate CTCs, depending on either the unique biophysical or biochemical properties of CTCs, or a combination of both (26). Different CTC enrichment methodologies have different sensitivities for CTC detection. ISET ${ }^{\circledR}$ stands for "Isolation of Epithelial Tumour Cells by Size", using microfiltration to enrich CTCs and then immunofluorescent staining of epithelial markers to identify CTCs. ISET ${ }^{\circledR}$ usually has a higher CTC detection rate in LC than CellSearch ${ }^{\circledR}$. Guibert et al. used ISET ${ }^{\circledR}$, and CTCs were detected in 89 out of 96 advanced NSCLC patients (93\%) (27). With the improvement of technologies, new CTC detection methods become more sensitive. Tong et al. used Cyttel, a negative immunomagnetic selection method, to detect CTCs in 127 patients with advanced NSCLC and the CTC detection rate was $84 \%$ (28). CytoSorter ${ }^{\circledR}$, a microfluidic-based CTC immunocapture platform, was employed in this study for CTC detection. One advantage about CytoSorter ${ }^{\circledR}$ is that any biotin-labeled antibody can be immobilized on the streptavidinfunctionalized CytoSorter ${ }^{\circledR}$ nanochip for capturing desired cells. Epithelial-to-mesenchymal transition (EMT) is a common phenomenon during cancer development (29). EMT causes the reduced expression of epithelial markers, such as EpCAM and cytokeratin, and enhanced expression of mesenchymal markers, such as vimentin, twist, snail and slug (30). Over-expression of vimentin in cancer cells is highly correlated with cancer progression, and EMT would lead to the translocation of vimentin from the intracellular region to the cell surface to become cell surface vimentin (CSV) (31). It is reported that CSV can be used as a target for capturing EMT and mesenchymal CTCs (32). Previous study has shown CTC detection rate with CSV antibody was higher than that with EpCAM antibody in breast and pancreatic cancers $(18,33)$. This study aimed to evaluate CSV-CTCs as a biomarker for LC. Our first result indicate CSV-CTCs can distinguish LC patients from BLD patients and HD. As suggested by the other studies and our results, CTCs can be used in general as a diagnostic biomarker for LC (19).

When CTC cut-off was set to 2 CTCs per $7.5 \mathrm{~mL}$ of blood, CSV-CTCs have a sensitivity and specificity of 0.67 and 0.87 , respectively, for diagnosing LC. The cut-off value is consistent with the previous finding in pancreatic cancer (18). Different CTC systems use different methodologies and thus have different sensitivities. Results from different CTC platforms are not comparable due to the different cut-off values caused by different sensitivities. Table 7 summarizes recent studies concerning the use of CTCs as a diagnostic tool for LC (15-17, 34-37). In spite of different CTC detection methods, these studies all come to the same conclusion that CTCs can be used as a diagnostic biomarker for LC. Among them, EpCAM based methods, such as CellSearch ${ }^{\circledR}$ or CellCollector, usually have a lower sensitivity due to the lower CTC detection rate. CSV-CTCs 
TABLE 6 | Correlation of serum tumor biomarkers with LC patients' clinicopathological characteristics.

\begin{tabular}{|c|c|c|c|c|c|c|c|c|c|c|c|c|c|}
\hline \multirow[t]{2}{*}{ Characteristics } & \multirow[t]{2}{*}{$\mathbf{n}$} & \multicolumn{2}{|c|}{ NSE (ng/mL) } & \multirow[t]{2}{*}{$P$} & \multicolumn{2}{|c|}{ CEA (ng/mL) } & \multirow[t]{2}{*}{$\boldsymbol{P}$} & \multicolumn{2}{|c|}{ CA125 (U/mL) } & \multirow[t]{2}{*}{$P$} & \multicolumn{2}{|c|}{ CA153 (U/mL) } & \multirow[t]{2}{*}{$\mathbf{P}$} \\
\hline & & Mean & Median & & Mean & Median & & Mean & Median & & Mean & Median & \\
\hline \multicolumn{14}{|l|}{ Gender } \\
\hline Male & 40 & 18.51 & 16.86 & 0.3019 & 28.48 & 4.04 & 0.4702 & 48.12 & 20.1 & 0.9643 & 23.37 & 12.19 & 0.4024 \\
\hline Female & 27 & 23.24 & 18.2 & & 111.65 & 3.08 & 55.08 & 17.74 & 30.56 & 14.28 & & & \\
\hline \multicolumn{14}{|l|}{ Age } \\
\hline$\geq 60$ & 36 & 21.07 & 18.05 & 0.0518 & 94.66 & 4.47 & 0.3392 & 58.33 & 25.25 & 0.2473 & 31.1 & 16.75 & 0.0291 \\
\hline$<60$ & 31 & 19.06 & 14.71 & & 24.06 & 2.59 & 42.31 & 16.58 & 20.65 & 10.04 & & & \\
\hline \multicolumn{14}{|l|}{ Histology } \\
\hline Adenocarcinoma & 56 & 20.41 & 17.05 & 0.6816 & 71.22 & 4.47 & 0.2395 & 55.88 & 18.44 & 0.9393 & 29.44 & 14.99 & 0.0081 \\
\hline Squamous & 11 & 19.5 & 18.94 & & 15.03 & 2.43 & 25.68 & 16.58 & 10.12 & 9.23 & & & \\
\hline \multicolumn{14}{|l|}{ Smoking History } \\
\hline Yes & 30 & 20.16 & 17.33 & 0.3993 & 28.28 & 3.965 & 0.6959 & 42.85 & 20.92 & 0.8352 & 20.02 & 11.44 & 0.2435 \\
\hline No & 37 & 20.3 & 17.17 & & 89.33 & 3.87 & 57.46 & 17.74 & 31.33 & 14.78 & & & \\
\hline \multicolumn{14}{|l|}{ TNM Stage } \\
\hline 1 & 21 & 13.59 & 13.37 & 0.0018 & 2.29 & 1.78 & $<0.0001$ & 11 & 9.53 & $<0.0001$ & 10.3 & 9.14 & $<0.0001$ \\
\hline$\|$ & 6 & 16.31 & 16.86 & & 3.15 & 2.1 & 16.35 & 8.67 & 14.78 & 14.86 & & & \\
\hline III & 13 & 19.81 & 18.94 & & 41.34 & 3.81 & 48.39 & 21.07 & 17.12 & 11.33 & & & \\
\hline IV & 27 & 24.92 & 20.17 & & 131.45 & 11.4 & 91.26 & 40.68 & 45.64 & 27.24 & & & \\
\hline \multicolumn{14}{|l|}{ Tumor Depth } \\
\hline $\mathrm{T} 1$ & 27 & 14.34 & 13.89 & 0.0065 & 107.9 & 2.53 & 0.052 & 18.66 & 13.16 & $<0.0001$ & 14.34 & 10.99 & 0.0024 \\
\hline $\mathrm{T} 2$ & 11 & 20.24 & 18.52 & & 7 & 3.81 & 18 & 11.68 & 15.83 & 11.55 & & & \\
\hline T3 & 16 & 20.18 & 17.19 & & 44.84 & 5.175 & 45.71 & 29.4 & 24.59 & 16.71 & & & \\
\hline T4 & 13 & 27.98 & 19.06 & & 34.26 & 10.08 & 152.2 & 96.68 & 61.94 & 33.53 & & & \\
\hline \multicolumn{14}{|c|}{ Lymph Node Involvement } \\
\hline NO & 30 & 14.86 & 14.71 & 0.0033 & 92.16 & 1.94 & 0.0011 & 17.39 & 9.995 & 0.0002 & 13.56 & 11.23 & 0.0335 \\
\hline N1 & 4 & 27.94 & 27.26 & & 9.063 & 8.95 & 32.23 & 27.35 & 35.67 & 25.22 & & & \\
\hline N2 & 19 & 22.87 & 18.25 & & 36.64 & 4.11 & 90.72 & 29 & 32.65 & 12.08 & & & \\
\hline N3 & 14 & 23.07 & 18.14 & & 46.89 & 21.87 & 74.11 & 68.26 & 42.14 & 16.94 & & & \\
\hline \multicolumn{14}{|c|}{ Lymph Node Metastasis } \\
\hline Yes & 37 & 23.55 & 19 & 0.001 & 37.54 & 8.7 & 0.0002 & 78.11 & 29.79 & $<0.0001$ & 36.57 & 16.9 & 0.008 \\
\hline No & 30 & 14.86 & 14.71 & & 92.16 & 1.94 & 17.39 & 9.995 & 13.56 & 11.23 & & & \\
\hline \multicolumn{14}{|c|}{ Distant Metastasis } \\
\hline $\mathrm{MO}$ & 40 & 16.32 & 15.85 & 0.0046 & 15.11 & 2.455 & $<0.0001$ & 23.7 & 11.68 & $<0.0001$ & 13.19 & 11.07 & $<0.0001$ \\
\hline M1 & 27 & 24.92 & 20.17 & & 131.5 & 11.4 & 91.26 & 40.68 & 45.64 & 27.24 & & & \\
\hline \multicolumn{14}{|c|}{ CSV Positive CTC Status } \\
\hline No Detected (0) & 9 & 13.34 & 11.64 & 0.0063 & 2.38 & 2.31 & 0.0191 & 20.23 & 14.89 & 0.1981 & 9.532 & 8.73 & 0.0067 \\
\hline Detected $(\geq 1)$ & 58 & 21.4 & 17.8 & & 71.25 & 4.47 & 55.68 & 22.65 & 28.86 & 14.53 & & & \\
\hline Positive $(\geq 2)$ & 40 & 21.55 & 17.18 & 0.2013 & 29.54 & 4.89 & 0.0202 & 62.52 & 25.27 & 0.0315 & 31.6 & 15.2 & 0.0279 \\
\hline Negative $(<2)$ & 27 & 15.98 & 15.75 & & 138.3 & 2.395 & 23.66 & 13.29 & 13.75 & 9.91 & & & \\
\hline
\end{tabular}

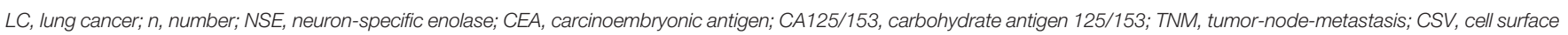
vimentin; CTC, circulating tumor cell. Bold values mean statistically significant. Bold values are all less than 0.05 .

TABLE 7 | Summary of studies concerning the diagnostic performance of CTCs in LC diagnosis.

\begin{tabular}{|c|c|c|c|c|c|c|c|c|}
\hline First author & Year & Methodology & No of patients (control) & Mean age (year) & Cut-off value & AUC & Sensitivity & Specificity \\
\hline Yu Y (35) & 2013 & FR PCR & $153(113)$ & 59.4 & 8.64 CTC units & 0.823 & 0.73 & 0.84 \\
\hline Chen X (24) & 2015 & CytoploRare (FR PCR) & $473(283)$ & 55.1 & 8.93 CTC units & 0.815 & 0.74 & 0.87 \\
\hline Li Y (25) & 2019 & NE-FISH & $174(90)$ & $\mathrm{N} / \mathrm{A}$ & CTCs $\geq 2 / 3.2 \mathrm{~mL}$ & 0.846 & 0.68 & 1 \\
\hline Duan GC (23) & 2020 & GILUPI CellCollector & $44(20)$ & 56 & CTCs $\geq 1$ & 0.715 & 0.53 & 0.9 \\
\hline
\end{tabular}

CTC, circulating tumor cells; $L C$, lung cancer; no, number; AUC, area under curve; N/A, not applicable; FR PCR, folate receptor ligand-targeted polymerase chain reaction; CD45, cluster of differentiation 45; CSV, cell surface vimentin. 
are usually CTCs that underwent EMT, representing the mesenchymal and the mixed types of CTCs. Our results show that CSV-CTCs can be used as a diagnostic biomarker for LC. As shown in Table 7, the diagnostic potency of the CSV based strategy is slightly better than the EpCAM based methods. Techniques using physical properties such as size difference or negative immuno-selection to enrich CTCs might have a higher CTC detection rate although with lower purity. High expression of FR alpha $(F R \alpha)$ is usually observed in LC, especially in adenocarcinoma. Therefore, FR based PCR has been proposed to detect CTC in LC, and the results are promising. FR based PCR method has a better sensitivity and specificity as shown in Table 7 (16, 35). In fact, in the Chinese expert consensus on lung cancer screening and management, it is suggested that FR PCR based CTC detection can be used in conjugation with medical imaging examination to enhance the diagnostic specificity of lung nodule diagnosis (38). Li et al. used immunolipid magnetic spheres conjugated with 3 different antibodies, epidermal growth factor receptor (EGFR), vimentin and folic acid (FA), to detect CTC in early stage NSCLC patients. Using 2 CTCs per $7.5 \mathrm{~mL}$ of blood as cut-off value, the positive rates of EGFR, vimentin and FA magnets used alone and in combination in LC patients were $65 \%, 33.3 \%, 93.3 \%$ and $100 \%$, respectively (39). It reconfirms that vimentin is not a good target to be used to capture CTCs in early stage LC. However, the combination of multiple targets, such as EpCAM, CSV, EGFR or FA, might be a good strategy to increase the CTC detection rate in early stage LC.

One major drawback of the CSV based method is that many false positives were found in patients with BLD. Although CSV positive cells were not detected in $\mathrm{HD}$, they were found in 10 out of 21 patients with BLD. For patients with BLD who were found to have CSV positive cells, $90 \%$ of patients had either inflammation diseases, fibrosis or other lung infections. The reason for the false positive is that CSV is not a tumor specific marker. In fact, most of the targets used for CTC detection are not tumor specific. CSV has been identified to participate in cell adhesion, migration and cellular signaling (21). Expression of CSV is not only seen in cells undergoing EMT, but also in cells infected with certain virus, and in activated lymphocytes, myofibroblasts and stellate cells (40-42). As inflammation and fibrosis are usually common observed in patients with BLD, the false positives might come from the activated lymphocytes and myofibroblasts. A counterstain of activated lymphocytes and myofibroblasts or use of a tumor specific marker should be applied to reduce false positives.

CSV-CTCs are correlated with LC patients' cancer stage, lymph node involvement and distant metastasis, which is consistent with previous findings that CTCs can reflect tumor burden in LC (15, 16). Our results surprisingly show that CSV-CTCs are correlated with LC patients' age. It could be that older patents recruited in this study had usually advanced tumors. If LC patients were first grouped by cancer stage, no significant correlation between CTCs and age would be found in each group (data not shown). CSV-CTC enumeration was not associated with tumor size. CTCs captured by CSV antibody was only one subtype of CTCs. As Li used 3 different antibodies to enrich CTC in LC and found that only CTC enumeration detected by the combined use of 3 antibodies was correlated with cancer stages (39). It suggests that the total CTCs, but not one subtype, may be correlated with tumor size. However, CSV-CTC positive rate was correlated with tumor size, lymph node involvement, distant metastasis, but not with cancer stage, which might be explained by the small sample size. It is believed that CTCs undergoing EMT survive better in circulation and have higher chance to colonize at distant site to form metastasis (43). Therefore, cancer patients with metastasis should have more EMT and mesenchymal CTCs, which is in line with our findings in this study that LC patients with lymph node or distant metastasis tend to have more CSV-CTCs, which might represent the CTC subtype with more invasive nature.

Serum tumor markers have been used extensively in daily practice for LC diagnosis. Therefore, lastly, we liked to compare the diagnostic potency of serum tumor markers and CTCs for LC and see whether the combination of serum tumor markers with CTCs would improve the diagnostic performance. Serum levels of NSE, CEA, CA125 or CA153 cannot distinguish LC patients from the control, indicating that serum tumor markers are not reliable markers for LC screening. Similar to the CSV-CTCs, serum tumor marker test generates many false positives in patients with BLD. However, in some extent, all serum tumor markers can somehow reflect LC patients' clinicopathological characteristics as shown in Table 6. Some serum tumor markers can even reflect the status of CSV-CTCs. Studies showed that a combination of several tumor markers can produce a higher sensitivity $(44,45)$. However, the best combination of tumor markers for diagnosing LC remains unknown. The AUC of the combined use of NSE, CEA, CA125 and CA153 is still smaller than that of CSV-CTCs alone (data not shown). Li et al. also found that the diagnostic sensitivity for LC yielded from the combination of four serum tumor markers, CEA, CA125, cytokeratin fragment 21-1 (CYFRA 21-1), and squamous cell carcinoma (SCC), was still lower than that achieved based on CTC counts alone (17). Conjugation of serum tumor markers with CSV-CTCs unexpectedly did not improve the diagnostic performance, which is contradictory to a previous finding that diagnostic performance for LC would be improved by combining CTCs with serum tumor markers (17). It could be explained by the different CTC populations captured in these two studies. While we detected the EMT CTCs, the other study detected total CTCs. Both CTC and serum tumor markers can generate false positive or negative result. Thus, they can only be used as a reference in practice. Biopsy should be still considered as the gold standard for disease confirmation.

Although it is reported that CSV can be used as a target to enrich CTCs in sarcoma, breast, pancreatic, prostate and gastric cancers $(18,32,33,46,47)$, the high false positive results in patients with BLD raised the question that whether CTCs captured by anti-CSV were truly tumor cells. One major limitation of this study is that we did not collect the captured CTCs for any downstream analysis to confirm its identity. Also sample size was limited. We might get more statistically significant results if more LC patients with different cancer stages were recruited in this study. 


\section{CONCLUSIONS}

Results of this study show that CSV-CTCs can be used as an acceptable biomarker for LC with a sensitivity and specificity of 0.67 and 0.87 , respectively. CSV-CTCs are positively correlated with lymph node and distant metastases, indicating that CSVCTCs represent the CTC subtype with more invasive nature. Still further research with larger patient population is needed to verify our findings.

\section{DATA AVAILABILITY STATEMENT}

The raw data supporting the conclusions of this article will be made available by the authors, without undue reservation.

\section{ETHICS STATEMENT}

This study followed the principles established in the Declaration of Helsinki and was approved by the ethics committee of the First Affiliated Hospital of Guangzhou Medical University. Written informed consent to the publication of their case details was obtained from each patient and healthy donor. The patients/ participants provided their written informed consent to participate in this study.

\section{REFERENCES}

1. Siegel RL, Miller KD, Jemal A. Cancer Statistics, 2019. CA Cancer J Clin (2019) 69(1):7-34. doi: 10.3322/caac.21551

2. Bray F, Ferlay J, Soerjomataram I, Siegel RL, Torre LA, Jemal A. Global Cancer Statistics 2018: GLOBOCAN Estimates of Incidence and Mortality Worldwide for 36 Cancers in 185 Countries. CA Cancer J Clin (2018) 68 (6):394-424. doi: 10.3322/caac.21492

3. Zheng RS, Sun KX, Zhang SW, Zhen HM, Zou XN, Chen R, et al. Report of Cancer Epidemiology in China. Zhonghua Zhong Liu Za Zhi (2019) 41(1):1928. doi: 10.3760/cma.j.issn.0253-3766.2019.01.005

4. Hirsch FR, Scagliotti GV, Mulshine JL, Kwon R, Curran WJ Jr, Wu YL, et al. Lung Cancer: Current Therapies and New Targeted Treatments. Lancet (2017) 389(10066):299-311. doi: 10.1016/S0140-6736(16)30958-8

5. Inage T, Nakajima T, Yoshino I, Yasufuku K. Early Lung Cancer Detection. Clin Chest Med (2018) 39(1):45-55. doi: 10.1016/j.ccm.2017.10.003

6. Wang X, Zhang Y, Sun L, Wang S, Nie J, Zhao W, et al. Evaluation of the Clinical Application of Multiple Tumor Marker Protein Chip in the Diagnostic of Lung Cancer. J Clin Lab Anal (2018) 32(8):e22565. doi: 10.1002/jcla.22565

7. Micalizzi DS, Maheswaran S, Haber DA. A Conduit to Metastasis: Circulating Tumor Cell Biology. Genes Dev (2017) 31(18):1827-40. doi: 10.1101/ gad.305805.117

8. Jiang SS, Deng B, Feng YG, Qian K, Tan QY, Wang RW. Circulating Tumor Cells Prior to Initial Treatment is an Important Prognostic Factor of Survival in non-Small Cell Lung Cancer: A Meta-Analysis and System Review. BMC Pulm Med (2019) 19(1):262. doi: 10.1186/s12890-019-1029-x

9. Maly V, Maly O, Kolostova K, Bobek V. Circulating Tumor Cells in Diagnosis and Treatment of Lung Cancer. In Vivo (2019) 33(4):1027-37. doi: 10.21873/ invivo. 11571

10. Chinniah C, Aguarin L, Cheng P, Decesaris C, Cutillo A, Berman AT, et al. Early Detection of Recurrence in Patients With Locally Advanced Non-SmallCell Lung Cancer Via Circulating Tumor Cell Analysis. Clin Lung Cancer (2019) 20(5):384-390.e2. doi: 10.1016/j.cllc.2019.04.011

\section{AUTHOR CONTRIBUTIONS}

Conception and design: XX, LW, XN W, SL and CZ. Acquisition of data: XX, LQ W, XN W, YQ, XL, ZX, ML, and MO. Analysis and interpretation of data: XX, LW, XW, W-HF, and CZ Z. Writing of the manuscript: XX and W-HF. All authors contributed to the article and approved the submitted version.

\section{FUNDING}

This study is supported by the following grants: Zhongnanshan Medical Foundation of Guangdong Province [ZNSA-2020003], Guangdong Science and Technology Program special projects [2020A1111350025], Wu Jieping Medical Foundation [320.6750.19088-8], and Beijing Bethune Charitable Foundation [BQE-TY-SSPC(5)-S-03].

\section{ACKNOWLEDGMENTS}

We would like to thank all the members of clinical laboratory of IMPROVE MEDICAL for the help in CTC detection. Special thanks to Dr. NG for reading this manuscript and valuable opinions.

11. Kapeleris J, Kulasinghe A, Warkiani ME, Vela I, Kenny L, O’Byrne K, et al. The Prognostic Role of Circulating Tumor Cells (Ctcs) in Lung Cancer. Front Oncol (2018) 8:311. doi: 10.3389/fonc.2018.00311

12. Hu Z, Ding J, Ma Z, Sun R, Seoane JA, Scott Shaffer J, et al. Quantitative Evidence for Early Metastatic Seeding in Colorectal Cancer. Nat Genet (2019) 51(7):1113-22. doi: 10.1038/s41588-019-0423-x

13. Agnoletto C, Corrà F, Minotti L, Baldassari F, Crudele F, Cook WJJ, et al. Heterogeneity in Circulating Tumor Cells: The Relevance of the Stem-Cell Subset. Cancers (Basel) (2019) 11(4):483. doi: 10.3390/cancers11040483

14. Kulasinghe A, Kapeleris J, Cooper C, Warkiani ME, O’Byrne K, Punyadeera C. Phenotypic Characterization of Circulating Lung Cancer Cells for Clinically Actionable Targets. Cancers (Basel) (2019) 11(3):380. doi: 10.3390/ cancers 11030380

15. Duan GC, Zhang XP, Wang HE, Wang ZK, Zhang H, Yu L, et al. Circulating Tumor Cells as a Screening and Diagnostic Marker for Early-Stage non-Small Cell Lung Cancer. Onco Targets Ther (2020) 13:1931-9. doi: 10.2147/ OTT.S241956

16. Li Y, Tian X, Gao L, Jiang X, Fu R, Zhang T, et al. Clinical Significance of Circulating Tumor Cells and Tumor Markers in the Diagnosis of Lung Cancer. Cancer Med (2019) 8(8):3782-92. doi: 10.1002/cam4.2286

17. Chen X, Zhou F, Li X, Yang G, Zhang L, Ren S, et al. Folate Receptor-Positive Circulating Tumor Cell Detected by LT-PCR-Based Method as a Diagnostic Biomarker for Non-Small-Cell Lung Cancer. J Thorac Oncol (2015) 10 (8):1163-71. doi: 10.1097/JTO.0000000000000606

18. Wei T, Zhang X, Zhang Q, Yang J, Chen Q, Wang J, et al. Vimentin-Positive Circulating Tumor Cells as a Biomarker for Diagnosis and Treatment Monitoring in Patients With Pancreatic Cancer. Cancer Lett (2019) 452:237-43. doi: 10.1016/j.canlet.2019.03.009

19. Huang H, Shi Y, Huang J, Wang X, Zhang R, Chen H. Circulating Tumor Cells as a Potential Biomarker in Diagnosis of Lung Cancer: A Systematic Review and Meta-Analysis. Clin Respir J (2018) 12(2):639-45. doi: 10.1111/crj.12573

20. Hayes DF, Cristofanilli M, Budd GT, Ellis MJ, Stopeck A, Miller MC, et al. Circulating Tumor Cells At Each Follow-Up Time Point During Therapy of Metastatic Breast Cancer Patients Predict Progression-Free and Overall 
Survival. Clin Cancer Res (2006) 12(14):4218-24. doi: 10.1158/10780432.CCR-05-2821

21. Cohen SJ, Punt C, Iannotti N, Saidman BH, Sabbath KD, Gabrail NY, et al. Relationship of Circulating Tumor Cells to Tumor Response, ProgressionFree Survival, and Overall Survival in Patients With Metastatic Colorectal Cancer. Clin Oncol (2008) 26:3213-21. doi: 10.1200/JCO.2007.15.8923

22. de Bono JS, Scher HI, Montgomery RB, Parker C, Miller MC, Tissing H, et al. Circulating Tumor Cells Predict Survival Benefit From Treatment in Metastatic Castration-Resistant Prostate Cancer. Clin Cancer Res (2008) 14 (19):6302-9. doi: 10.1158/1078-0432.CCR-08-0872

23. Krebs MG, Sloane R, Priest L, Lancashire L, Hou JM, Greystoke A, et al. Evaluation and Prognostic Significance of Circulating Tumor Cells in Patients With non-Small-Cell Lung Cancer. J Clin Oncol (2011) 29(12):1556-63. doi: 10.1200/JCO.2010.28.7045

24. Marchetti A, Del Grammastro M, Felicioni L, Malatesta S, Filice G, Centi I, et al. Assessment of EGFR Mutations in Circulating Tumor Cell Preparations From NSCLC Patients by Next Generation Sequencing: Toward a Real-Time Liquid Biopsy for Treatment. PloS One (2014) 9(8):e103883. doi: 10.1371/ journal.pone. 0103883

25. Ilie M, Szafer-Glusman E, Hofman V, Long-Mira E, Suttmann R, Darbonne W, et al. Expression of MET in Circulating Tumor Cells Correlates With Expression in Tumor Tissue From Advanced-Stage Lung Cancer Patients. Oncotarget (2017) 8(16):26112-21. doi: 10.18632/oncotarget.15345

26. Shen Z, Wu A, Chen X. Current Detection Technologies for Circulating Tumor Cells. Chem Soc Rev (2017) 46(8):2038-56. doi: 10.1039/C6CS00803H

27. Guibert N, Delaunay M, Lusque A, Boubekeur N, Rouquette I, Clermont E, et al. Pd-L1 Expression in Circulating Tumor Cells of Advanced non-Small Cell Lung Cancer Patients Treated With Nivolumab. Lung Cancer (2018) 120:108-12. doi: 10.1016/j.lungcan.2018.04.001

28. Tong B, Xu Y, Zhao J, Chen M, Xing J, Zhong W, et al. Prognostic Significance of Circulating Tumor Cells in non-Small Cell Lung Cancer Patients Undergoing Chemotherapy. Oncotarget (2017) 8(49):86615-24. doi: 10.18632/oncotarget.21255

29. Brabletz T, Kalluri R, Nieto MA, Weinberg RA. EMT in Cancer. Nat Rev Cancer (2018) 18(2):128-34. doi: 10.1038/nrc.2017.118

30. Satelli A, Mitra A, Brownlee Z, Xia X, Bellister S, Overman MJ, et al. Epithelial-Mesenchymal Transitioned Circulating Tumor Cells Capture for Detecting Tumor Progression. Clin Cancer Res (2015) 21(4):899-906. doi: 10.1158/1078-0432.CCR-14-0894

31. Satelli A, Li S. Vimentin in Cancer and its Potential as a Molecular Target for Cancer Therapy. Cell Mol Life Sci (2011) 68(18):3033-46. doi: 10.1007/ s00018-011-0735-1

32. Satelli A, Mitra A, Cutrera JJ, Devarie M, Xia X, Ingram DR, et al. Universal Marker and Detection Tool for Human Sarcoma Circulating Tumor Cells. Cancer Res (2014) 74(6):1645-50. doi: 10.1158/0008-5472.CAN-13-1739

33. Satelli A, Brownlee Z, Mitra A, Meng QH, Li S. Circulating Tumor Cell Enumeration With a Combination of Epithelial Cell Adhesion Molecule- and Cell-Surface Vimentin-Based Methods for Monitoring Breast Cancer Therapeutic Response. Clin Chem (2015) 61(1):259-66. doi: 10.1373/ clinchem.2014.228122

34. Tanaka F, Yoneda K, Kondo N, Hashimoto M, Takuwa T, Matsumoto S, et al. Circulating Tumor Cell as a Diagnostic Marker in Primary Lung Cancer. Clin Cancer Res (2009) 15(22):6980-6. doi: 10.1158/1078-0432.CCR-09-1095

35. Yu Y, Chen Z, Dong J, Wei P, Hu R, Zhou C, et al. Folate Receptor-Positive Circulating Tumor Cells as a Novel Diagnostic Biomarker in non-Small Cell Lung Cancer. Transl Oncol (2013) 6(6):697-702. doi: 10.1593/tlo.13535
36. Chen YY, Xu GB. Effect of Circulating Tumor Cells Combined With Negative Enrichment and CD45-FISH Identification in Diagnosis, Therapy Monitoring and Prognosis of Primary Lung Cancer [Published Correction Appears in Med Oncol. 2015 Jul;32(7):190]. Med Oncol (2014) 31(12):240. doi: 10.1007/ s12032-014-0240-0

37. Fiorelli A, Accardo M, Carelli E, Angioletti D, Santini M, Di Domenico M. Circulating Tumor Cells in Diagnosing Lung Cancer: Clinical and Morphologic Analysis. Ann Thorac Surg (2015) 99(6):1899-905. doi: 10.1016/j.athoracsur.2014.11.049

38. UCOM, Union Of Chinese Oncology Management. Consensus of Chinese Experts on Lung Cancer Screening and Management. Guo Ji Hu Xi Za Zhi (2019) 39(21):1604-15. doi: 10.3760/cma.j.issn.1673-436x.2019.21.002

39. Li G, Wang Y, Tan G, Liu Y, Xu Z, Feng H, et al. Preliminary Study on Detection of Circulating Tumor Cells in Lung Cancer by EGFR/Vimentin/ Folic Acid Magnetic Sphere. Zhongguo Fei Ai Za Zhi (2020) 10:3779. doi: 10.3779/j.issn.1009-3419.2020.103.05

40. Yu YT, Chien SC, Chen IY, Lai CT, Tsay YG, Chang SC, et al. Surface Vimentin is Critical for the Cell Entry of SARS-Cov. J BioMed Sci (2016) 23:14. doi: 10.1186/s12929-016-0234-7

41. Moisan E, Girard D. Cell Surface Expression of Intermediate Filament Proteins Vimentin and Lamin B1 in Human Neutrophil Spontaneous Apoptosis. J Leukoc Biol (2006) 79(3):489-98. doi: 10.1189/jlb.0405190

42. Song I, Ise H. Development of a Gene Delivery System of Oligonucleotides for Fibroses by Targeting Cell-Surface Vimentin-Expressing Cells With NAcetylglucosamine-Bearing Polymer-Conjugated Polyethyleneimine. Polymers (Basel) (2020) 12(7):1508. doi: 10.3390/polym12071508

43. Jie XX, Zhang XY, Xu CJ. Epithelial-to-Mesenchymal Transition, Circulating Tumor Cells and Cancer Metastasis: Mechanisms and Clinical Applications. Oncotarget (2017) 8(46):81558-71. doi: 10.18632/oncotarget.18277

44. Wu H, Wang Q, Liu Q, Zhang Q, Huang Q, Yu Z. The Serum Tumor Markers in Combination for Clinical Diagnosis of Lung Cancer. Clin Lab (2020) 66(3). doi: 10.7754/Clin.Lab.2019.190533

45. Plebani M, Basso D, Navaglia F, De Paoli M, Tommasini A, Cipriani A. Clinical Evaluation of Seven Tumour Markers in Lung Cancer Diagnosis: can Any Combination Improve the Results? Br J Cancer (1995) 72(1):170-3. doi: 10.1038/bjc.1995.296

46. Satelli A, Batth I, Brownlee Z, Mitra A, Zhou S, Noh H, et al. EMT Circulating Tumor Cells Detected by Cell-Surface Vimentin are Associated With Prostate Cancer Progression. Oncotarget (2017) 8(30):49329-37. doi: 10.18632/oncotarget.17632

47. Liu M, Wang R, Sun X, Liu Y, Wang Z, Yan J, et al. Prognostic Significance of PD-L1 Expression on Cell-Surface Vimentin-Positive Circulating Tumor Cells in Gastric Cancer Patients. Mol Oncol (2020) 14(4):865-81. doi: $10.1002 / 1878-0261.12643$

Conflict of Interest: W-HF is currently an employee of Watson Biotech.

The remaining authors declare that the research was conducted in the absence of any commercial or financial relationships that could be construed as a potential conflict of interest.

Copyright $\odot 2021$ Xie, Wang, Wang, Fan, Qin, Lin, Xie, Liu, Ouyang, Li and Zhou. This is an open-access article distributed under the terms of the Creative Commons Attribution License (CC BY). The use, distribution or reproduction in other forums is permitted, provided the original author(s) and the copyright owner(s) are credited and that the original publication in this journal is cited, in accordance with accepted academic practice. No use, distribution or reproduction is permitted which does not comply with these terms. 